\title{
Prevalência de Hipertensão Arterial Sistêmica e Diabetes Mellitus em Indivíduos com CoVID-19: Um Estudo Retrospectivo de Óbitos em Pernambuco, Brasil
}

\author{
Prevalence of Systemic Arterial Hypertension and Diabetes Mellitus in Individuals with COVID-19: A \\ Retrospective Study of Deaths in Pernambuco, Brazil
}

Lucas Gomes Santos, ${ }^{1}$ Jussara Almeida de Oliveira Baggio, ${ }^{1}$ Thiago Cavalcanti Leal, ${ }^{1}$ Francisco A. Costa, ${ }^{1}$ Tânia Rita Moreno de Oliveira Fernandes, ${ }^{2}$ Regicley Vieira da Silva, ${ }^{1}$ Anderson Armstrong, ${ }^{2}$ Rodrigo Feliciano Carmo, ${ }^{2}$ Carlos Dornels Freire de Souza ${ }^{10}$

Universidade Federal de Alagoas - Curso de Medicina, ${ }^{1}$ Maceió, AL - Brasil

Universidade Federal do Vale do São Francisco - Medicina, ${ }^{2}$ Petrolina, PE - Brasil

\section{Resumo}

Hipertensão arterial sistêmica (HAS) e diabetes mellitus (DM) são dois dos principais fatores de risco para a mortalidade por COVID-19.

Descrever a prevalência e o perfil clínico-epidemiológico de óbito por COVID-19 ocorridos em Pernambuco, Brasil, entre 12 de março e 14 de maio de 2020 entre pacientes que possuíam hipertensão arterial sistêmica e/ou diabetes mellitus como doenças prévias.

Estudo observacional transversal. Foram analisadas as seguintes variáveis: município de procedência, sexo, faixa etária, tempo entre o início dos sinais/sintomas e o óbito, sinais/sintomas, tipo de comorbidades e hábitos de vida. Variáveis categóricas foram descritas por meio de frequências e variáveis contínuas por meio de medidas de tendência central e de dispersão. Os testes de Mann-Whitney e Kruskal-Wallis foram utilizados.

Dos 1.276 registros incluídos no estudo, 410 apresentavam HAS e/ou DM. A prevalência de HAS foi $26,5 \%(n=338)$ e de DM foi 19,7\% $(n=252)$. Dos registros, $158(12,4 \%)$ eram de pacientes que possuíam somente HAS, 72 (5,6\%) somente DM e $180(14,1 \%)$ apresentavam HAS e DM. Dos indivíduos com HAS, 53,3\% apresentavam DM e 71,4\% dos diabéticos apresentam HAS. A mediana (em dias) do tempo entre o início dos sinais/sintomas e o desfecho óbito foi 8,0 (IIQ 9,0), sem diferença significativa entre os grupos de comorbidades $(p=0,633)$, sexo $(p=0,364)$ e faixa etária $(p=0,111)$. Observou-se maior prevalência de DM e HAS na população masculina (DM - 61,3\% eram homens e 38,9\%

\section{Palavras-chave}

Coronavírus-19; SARS-CoV-19; Pandemia; Hipertensão/ complicações; Diabetes Mellitus/complicações; Fatores de Risco/prevenção e controle; Idoso; Prevalência.

Correspondência: Carlos Dornels Freire de Souza •

Universidade Federal de Alagoas - Campus Arapiraca - Medicina

Av. Manoel Severino Barbosa, s/n. CEP 57309-005, Arapiraca, AL - Brasil

E-mail: carlos.freire@arapiraca.ufal.br

Artigo recebido em 05/06/2020, revisado em 23/10/2020,

aceito em 11/11/2020

DOI: https://doi.org/10.36660/abc.20200885 mulheres; HAS - 53,2\% eram homens e 46,8\% mulheres). Os sinais/sintomas mais frequentes foram dispneia $(74,1 \%$; $\mathrm{n}=304)$, tosse $(72,2 \% ; \mathrm{n}=296)$, febre $(68,5 \% ; n=281)$ e saturação de O2<95\% (66,1\%; $n=271)$. Dos hipertensos, $73,3 \%(n=100)$ apresentavam outras comorbidades/fatores de risco associados, e 54,2\% $(n=39)$ dos diabéticos apresentavam outras comorbidades/fatores de risco associados. Destacaramse as cardiopatias $(19,5 \% ; n=80)$, obesidade $(8,3 \% ; n=34)$, doença respiratória prévia $(7,3 \% ; n=30)$ e nefropatia $(7,8 \%$; $n=32)$. A prevalência de tabagismo foi $8,8 \%(n=36)$ e de etilismo alcançou 3,4\% $(n=14)$.

O estudo mostrou que a prevalência de HAS foi superior à prevalência de DM nos indivíduos que foram a óbito por COVID-19. Em idosos, a prevalência foi superior à observada em indivíduos não idosos.

\section{Introdução}

Em dezembro de 2019, as entidades sanitárias da província de Hubei, na República popular da China, identificaram e relataram à Organização Mundial da Saúde (OMS) um surto de uma pneumonia com agente etiológico até então desconhecido. ${ }^{1}$ No início de janeiro, o vírus SARSCoV-2 (Severe Acute Respiratory Syndrome Coronavírus 2) foi identificado e a doença foi denominada COVID-19 (Coronavirus Disease 2019). ${ }^{2}$

Em 4 de agosto de 2020, a doença já havia infectado um total de 18.316.072 pessoas e causado a morte de 694.715. EUA, Brasil e Índia ocupam as primeiras posições em número de doentes. ${ }^{3}$ No Brasil, o primeiro caso foi confirmado no dia 26 de fevereiro na cidade de São Paulo. Entre essa data e 4 de agosto de 2020, o país somou 2.750.249 infectados e 94.665 óbitos. $^{4}$

Devido ao impacto global causado pela pandemia, existe uma urgência na produção de conhecimento acerca do novo coronavírus. A caracterização das pessoas infectadas é essencial para o planejamento do combate à doença e da retomada econômica. Desde o começo da pandemia, diversos estudos foram publicados com esse intuito, e mostraram que a doença afeta de forma mais grave principalmente pessoas idosas com presença de comorbidades. ${ }^{5,6}$ A hipertensão arterial sistêmica (HAS) e o diabetes mellitus (DM) são as comorbidades mais frequentes nas pessoas que foram a óbito 
e sua fisiopatologia parece favorecer o desenvolvimento de quadros mais graves. ${ }^{7-9}$

Com a doença ainda em expansão em território brasileiro, é importante entender as características das pessoas infectadas no país e também em diferentes estados, devido ao tamanho continental e as diferenças socioeconômicas presentes no Brasil. ${ }^{10} \mathrm{O}$ estado de Pernambuco foi particularmente afetado, com registro de 98.833 casos e 6.717 óbitos em 4 de agosto de $2020 .{ }^{11}$

Dessa forma, o presente estudo teve como principal objetivo descrever a prevalência e o perfil clínico-epidemiológico de óbitos por COVID-19 ocorridos em Pernambuco entre 12 de março e 14 de maio de 2020, entre pacientes que possuíam hipertensão arterial sistêmica e/ou diabetes mellitus como doenças prévias.

\section{Métodos}

Trata-se de um estudo observacional transversal, envolvendo todos os óbitos por COVID-19 notificados em Pernambuco entre 12 de março e 14 de maio de 2020, entre pacientes que possuíam HAS e DM como doença de base. No estudo, foram analisadas as seguintes variáveis: sexo, faixa etária, tempo entre o início dos primeiros sintomas e o óbito, sinais/sintomas, a quantidade e o tipo de comorbidades associadas, além da HAS e do DM e hábitos de vida (tabagismo e etilismo). Os dados foram obtidos da página eletrônica de monitoramento da COVID-19 do estado de Pernambuco (https://dados.seplag. pe.gov.br/apps/corona.html) em 15 de maio de 2020. Após a coleta, o banco de dados passou por ajustes das variáveis, que consistiu na adequação dos sinais/sintomas e comorbidades e exclusão de registros inconsistentes.

Para a análise estatística, inicialmente, as variáveis categóricas foram descritas por meio de frequências (absolutas e relativas) e as variáveis contínuas por meio de medidas de tendência central e de dispersão. Para a comparação do tempo de início dos sintomas e o óbito entre os sexos feminino e masculino, utilizou-se o teste de Mann-Whitney, e entre as faixas etárias e grupo de comorbidades, utilizou-se o teste de Kruskal-Wallis com a aplicação posterior de teste post-hoc. Considerou-se intervalo de confiança de 95\% e significância de 5\%.

As análises foram realizadas com o auxílio do software SPSS versão 24.0 (IBM Corporation). Por utilizar dados de domínio público, nos quais não é possível a identificação dos indivíduos, este estudo dispensou a aprovação pelo Comitê de Ética em Pesquisa.

\section{Resultados}

Até o dia 14 de maio, constavam 1461 óbitos no banco de dados analisado do estado de Pernambuco. Desses casos, foram excluídos 185 pela baixa qualidade dos dados (ausência e/ou inconsistência entre as variáveis), restando 1.276 óbitos. Desses registros, 338 (26,48\%) apresentavam HAS e 252 $(19,74 \%)$ apresentavam DM como doenças de base: 158 $(12,4 \%)$ possuíam apenas HAS, $72(5,6 \%)$ apenas DM e $180(14,1 \%)$ HAS+DM. Dos indivíduos com HAS, 53,3\% apresentavam DM e 71,4\% dos diabéticos apresentavam HAS.

Os óbitos por COVID-19 tendo a HAS como doença de base foram registrados em 56 municípios, com destaque para Recife $(n=141)$, Jaboatão dos Guararapes $(n=27)$, Paulista $(n=27)$ e Olinda $(n=17)$, totalizando $62,72 \%(n=212)$ dos óbitos do estado. Já os óbitos por COVID-19 tendo o DM como doença de base foram registrados em 49 municípios, com destaque para Recife $(n=104)$, Jaboatão dos Guararapes $(n=21)$, Olinda $(n=13)$, Cabo de Santo Agostinho $(n=12)$ e Paulista $(n=12)$, na região metropolitana do Recife. Esses quatro municípios somaram $64,28 \%(n=162)$ dos óbitos.

A mediana (em dias) do tempo entre o início dos sinais/ sintomas e o desfecho óbito foi 8,0 (IIQ 9,0), sem diferença significativa entre os grupos de comorbidades $(p=0,633)$, sexo $(p=0,364)$, faixa etária $(p=0,111)$ e na comparação entre idosos e não idosos $(p=0,257)$ (Figura 1). Quanto ao perfil clínico e epidemiológico, observou-se distribuição homogênea entre os sexos no grupo geral $(n=410)$. No entanto, a análise desagregada mostrou maior prevalência de DM e HAS na população masculina (DM - 61,3\% eram homens e 38,9\% mulheres; HAS - 53,2\% eram homens e 46,8\% mulheres). Por outro lado, ao considerar apenas indivíduos com as duas comorbidades, observou-se predomínio de mulheres (53,3\%) (Tabela 1).

Destacou-se ainda a proporção de idosos na população estudada (73,4\% possuíam 60 anos ou mais; $n=301)$. Desses, 85,7\% $(n=258)$ apresentavam HAS, 59,5\% $(n=179)$ apresentavam DM e $45,2 \%(n=136)$ apresentavam as duas comorbidades. Os sinais/sintomas mais frequentes foram dispneia $(74,1 \% ; n=304)$, tosse $(72,2 \% ; n=296)$, febre $(68,5 \% ; n=281)$ e saturação de $02<95 \%(66,1 \% ; n=271)$ (Tabela 1).

No que concerne às comorbidades/fatores de risco associados, observou-se que $73,3 \%(n=100)$ dos hipertensos e $54,2 \%(n=39)$ dos diabéticos apresentavam outras comorbidades/fatores de risco associados. No grupo com HAS+DM, esse percentual foi de 54,4\% $(n=141)$. Dentre as comorbidades mais comuns, destacaram-se: cardiopatias $(19,5 \% / n=80)$, obesidade $(8,3 \% ; n=34)$, doença respiratória prévia $(7,3 \% ; n=30)$ e nefropatia $(7,8 \% ; n=32)$. A prevalência de tabagismo (atual ou pregresso) alcançou $8,8 \%(n=36)$ e o etilismo (atual ou pregresso) alcançou 3,4\% ( $n=14)$ (Tabela 1).

\section{Discussão}

A concentração dos óbitos descritos no presente estudo se concentra em municípios de maior porte (Recife e Jaboatão dos Guararapes) e pode relacionar-se ao número de indivíduos expostos e à circulação de pessoas, já que essas são as duas cidades mais populosas do estado. Soma-se a este fato a composição etária da população e da elevada prevalência de doenças crônicas não transmissíveis. ${ }^{12}$ Além disso, a disseminação da COVID-19 em Pernambuco parece seguir o padrão de outros países: a partir de grandes centros urbanos, se dissemina para cidades médias e pequenas. ${ }^{13}$

No que se refere à faixa etária, nota-se que a maior parte dos óbitos ocorreu em pessoas acima de 60 anos, principalmente na faixa etária de 70 a 79 anos, similar ao que tem sido observado em outros países previamente afetados pela pandemia. ${ }^{7,8} \mathrm{O}$ perfil de comorbidades da população brasileira também é um fator a ser levado em consideração. 


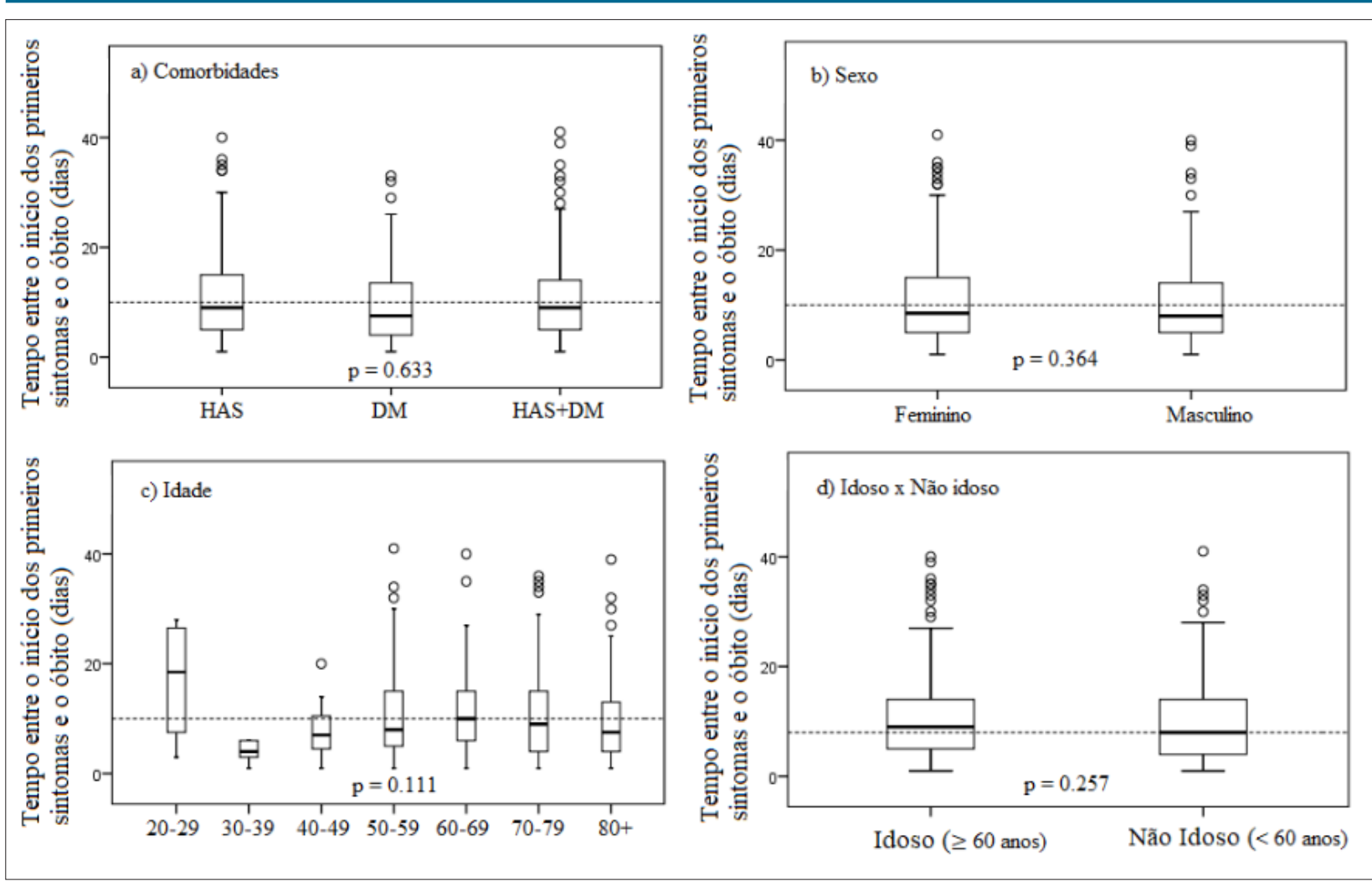

Figura 1 - Boxplot do tempo entre o início dos primeiros sintomas e óbito por COVID-19 em individuos com hipertensão arterial sistêmica e/ou diabetes mellitus. Pernambuco, Brasil.

A prevalência de DM é de 9,4\% na população geral e se torna ainda mais significante com o aumento da idade, cuja prevalência é de 22,6\% na população maior de 60 anos. ${ }^{14}$ Já a prevalência de HAS é de cerca de 24,0\%, alcançando $60,9 \%$ na população idosa. ${ }^{15}$ Indivíduos com HAS e DM prévio apresentam maior probabilidade de desenvolverem quadros mais graves da COVID-19, por vezes fatais. ${ }^{16}$

Além da idade, o sexo é outra característica relevante. Em revisão realizada por $\mathrm{Li}$ et al. ${ }^{17}$ na China, cerca de $60 \%$ dos infectados pelo SARS-CoV-2 eram homens. Resultados semelhantes foram apresentados por Zhou et al. ${ }^{8}$ tanto nos sobreviventes ( $59 \%$ eram homens) quanto nos indivíduos que foram a óbito (70\% homens), percentual superior ao observado em nosso estudo. A relação entre o sexo e a COVID-19 ainda não está elucidada, porém o pior desfecho no sexo masculino pode estar relacionado ao maior número de comorbidades presentes nos homens ou uma resposta do sistema imune diferente da observada na população feminina. ${ }^{17}$

O tempo entre o início dos sintomas e o óbito foi menor do que o descrito previamente na literatura (18,5 dias). ${ }^{6}$ No Brasil, a presença de comorbidades cardiovasculares pode reduzir o tempo de vida em até quatro dias. ${ }^{18}$ Entretanto, o resultado do nosso estudo pode estar subestimado, pois é necessário considerar uma possível dificuldade em reconhecer os primeiros sintomas, sobretudo nos indivíduos com condições socioeconômicas precárias e com nível educacional baixo. Além disso, o viés de memória é uma limitação dessa variável.
Em Pernambuco, 43,9\% dos óbitos investigados apresentavam HAS e DM simultaneamente. Em investigação realizada na cidade de Nova York envolvendo pacientes hospitalizados, as comorbidades mais frequentes foram HAS (56,6\%), obesidade $(41,7 \%)$ e DM $(33,8 \%)$, respectivamente. ${ }^{7}$ Essas comorbidades também foram descritas como as mais frequentes em diferentes investigações. ${ }^{8,19,20}$ A prevalência dessas doenças tem variado entre os países: na China, por exemplo, a presença dessas doenças é inferior à observada em países como Itália e EUA. ${ }^{21}$

Até o momento, sabe-se que o vírus SARS-CoV-2 liga-se à enzima conversora de angiotensina 2 (ECA-2), diminuindo a atividade desse tipo de receptor e levando a aumento da permeabilidade vascular. ${ }^{22}$ Este receptor tem uma expressão maior nos pulmões e no coração, sendo fundamental para o funcionamento desses sistemas. ${ }^{23} \mathrm{Em}$ pacientes com HAS e DM, existe um aumento desse tipo de receptor em comparação com a população saudável, o que pode levar ao desenvolvimento de quadros mais severos da doença. ${ }^{23}$ Além do mais, o SARS-CoV-2 promove lesão endotelial principalmente nos capilares pulmonares, promovendo um estado pró-coagulação, estado vascular inflamatório e de infiltrado celular, o que pode justificar quadros mais graves em pacientes com DM e obesos. ${ }^{24-26}$

Adicionalmente, os indivíduos com DM parecem apresentar uma resposta ao SARS-CoV-2 com grandes volumes por interferon (IFN) e resposta tardia de Th1/Th17, 
Tabela 1 - Caracterização clínica e epidemiológica dos óbitos por COVID-19 entre pacientes que apresentavam hipertensão arterial sistêmica e/ou diabetes mellitus como doença prévia. Pernambuco, Brasil

\begin{tabular}{|c|c|c|c|c|c|c|c|c|}
\hline \multirow{2}{*}{$\begin{array}{l}\text { Variável } \\
\text { Sexo }\end{array}$} & \multicolumn{2}{|c|}{ HAS $(n=158)$} & \multicolumn{2}{|c|}{$\mathrm{DM}(\mathrm{n}=72)$} & \multicolumn{2}{|c|}{$H A S+D M(n=180)$} & \multicolumn{2}{|c|}{ Total $(n=410)$} \\
\hline & $\mathbf{n}$ & $\%$ & n & $\%$ & $\mathbf{n}$ & $\%$ & $\mathbf{n}$ & $\%$ \\
\hline Feminino & 74 & 46,8 & 28 & 38,9 & 96 & 53,3 & 198 & 48,3 \\
\hline Masculino & 84 & 53,2 & 44 & 61,1 & 84 & 46,7 & 212 & 51,7 \\
\hline \multicolumn{9}{|l|}{ Idade $^{1}$} \\
\hline $20-29$ & 1 & 0,6 & 2 & 2,8 & 1 & 0,6 & 4 & 1,0 \\
\hline $30-39$ & 2 & 1,3 & 1 & 1,4 & 2 & 1,1 & 5 & 1,2 \\
\hline $40-49$ & 9 & 5,7 & 4 & 5,6 & 10 & 5,6 & 23 & 5,6 \\
\hline $50-59$ & 24 & 15,2 & 22 & 30,6 & 31 & 17,2 & 77 & 18,8 \\
\hline $60-69$ & 40 & 25,3 & 13 & 18,1 & 41 & 22,8 & 94 & 22,9 \\
\hline $70-79$ & 38 & 24,1 & 22 & 30,6 & 55 & 30,6 & 115 & 28,0 \\
\hline $80+$ & 44 & 27,8 & 8 & 11,1 & 40 & 22,2 & 92 & 22,4 \\
\hline \multicolumn{9}{|l|}{ Sinais/sintomas ${ }^{2}$} \\
\hline Dispneia & 111 & 70,3 & 54 & 75,0 & 139 & 77,2 & 304 & 74,1 \\
\hline Tosse & 117 & 74,1 & 51 & 70,8 & 128 & 71,1 & 296 & 72,2 \\
\hline Febre & 110 & 69,6 & 46 & 63,9 & 125 & 69,4 & 281 & 68,5 \\
\hline Saturação < $95 \%$ & 99 & 62,7 & 57 & 79,2 & 115 & 63,9 & 271 & 66,1 \\
\hline Dor de Garganta & 13 & 8,2 & 12 & 16,7 & 17 & 9,4 & 42 & 10,2 \\
\hline Diarreia & 6 & 3,8 & 4 & 5,6 & 11 & 6,1 & 21 & 5,1 \\
\hline Vômito & 4 & 2,5 & 5 & 6,9 & 6 & 3,3 & 15 & 3,7 \\
\hline Mialgia & 5 & 3,2 & 0 & 0,0 & 8 & 4,4 & 13 & 3,2 \\
\hline Astenia & 6 & 3,7 & 1 & 1,4 & 4 & 2,2 & 11 & 2,7 \\
\hline \multicolumn{9}{|l|}{$\begin{array}{l}N^{0} \text { Comorbidades além da } \\
\text { comorbidade de base }^{3}\end{array}$} \\
\hline Uma comorbidade & 58 & 36,7 & 33 & 45,8 & 0 & 0,0 & 91 & 22,2 \\
\hline Duas comorbidades & 68 & 43,0 & 28 & 38,9 & 82 & 45,6 & 178 & 43,4 \\
\hline Três ou mais & 32 & 20,3 & 11 & 15,3 & 98 & 54,4 & 141 & 34,4 \\
\hline \multicolumn{9}{|l|}{ Comorbidades } \\
\hline Cardiopatia & 25 & 15,8 & 19 & 26,4 & 36 & 20,0 & 80 & 19,5 \\
\hline Obesidade & 14 & 8,9 & 5 & 6,9 & 15 & 8,3 & 34 & 8,3 \\
\hline Doença respiratória prévia & 16 & 10,1 & 3 & 4,2 & 11 & 6,1 & 30 & 7,3 \\
\hline Nefropatia & 14 & 8,9 & 3 & 4,2 & 15 & 8,3 & 32 & 7,8 \\
\hline Doença neurológica prévia & 13 & 8,2 & 6 & 8,3 & 8 & 4,4 & 27 & 6,6 \\
\hline Câncer & 5 & 3,2 & 1 & 1,4 & 6 & 3,3 & 12 & 2,9 \\
\hline \multicolumn{9}{|l|}{ Hábitos de vida } \\
\hline Tabagismo atual & 12 & 7,6 & 3 & 4,2 & 8 & 4,4 & 23 & 5,6 \\
\hline Tabagismo pregresso & 7 & 4,4 & 2 & 2,8 & 4 & 2,2 & 13 & 3,2 \\
\hline Etilismo atual & 5 & 3,2 & 2 & 2,8 & 4 & 2,2 & 11 & 2,7 \\
\hline Etilismo pregresso & 2 & 1,3 & 0 & 0,0 & 1 & 0,6 & 3 & 0,7 \\
\hline
\end{tabular}

${ }^{1}$ Sem registros em individuos com idade inferior a 20 anos. ${ }^{2}$ Sinais/sintomas e comorbidades com frequência $<2,0 \%$ foram suprimidos. ${ }^{3}$ Comorbidades de base são HAS e DM. HAS: Hipertensão arterial sistêmica; DM: Diabetes mellitus. 
contribuindo para uma resposta inflamatória mais intensa. ${ }^{27}$ Um recente estudo in vitro demonstrou que a concentração de glicose em monócitos estava relacionada a um aumento da replicação viral e produção de citocinas pró-inflamatórias. ${ }^{28}$

O somatório de diferentes comorbidades em um mesmo indivíduo pode resultar em amplificação da resposta inflamatória e favorecer a rápida progressão e/ou agravamento do quadro clínico, reduzindo a sobrevida dos pacientes. ${ }^{27,28}$ Nessa análise, as comorbidades mais prevalentes associadas ao DM e à HAS foram cardiopatia não especificada e obesidade. Essas comorbidades também foram observadas em estudo conduzido em Nova York, no qual 18,0\% dos indivíduos possuíam cardiopatia e $41,7 \%$, obesidade. ${ }^{5}$ Atualmente, a alta prevalência de obesidade tem sido um grave problema de saúde pública na maioria dos países, inclusive no Brasil.

Os hábitos de vida, tais como tabagismo e etilismo, também podem agravar ainda mais esse risco quando relacionado à COVID-19. Indivíduos fumantes, quando infectados, apresentam 3,5 vezes mais chance de desenvolver formas mais agressivas da doença do que não fumantes. ${ }^{29}$ Por conseguinte, a prática aumenta o risco de lesão pulmonar culminando em bronquiolite respiratória crônica, diversos tipos de pneumonia, cânceres e enfisema pulmonar, ${ }^{30}$ que individualmente são fatores de risco para o SARS-CoV-2 e, em conjunto, diminuem a função pulmonar, aumentando a susceptibilidade ao vírus.

Sobre o consumo de bebidas alcoólicas, entende-se que, quando realizado de forma crônica, resulta em aumento das respostas pró-inflamatórias e redução das defesas antiinflamatórias intermediadas pelas citocinas. ${ }^{31}$ Associado a isso, o sistema imunológico como um todo é prejudicado com a prática do etilismo por reduzir a capacidade de combater agentes infecciosos através da imunidade inata e adaptativa, expondo de forma mais agressiva os contaminados pelo SARS-CoV-2. ${ }^{31}$

Ainda não se conhece os efeitos acumulados das comorbidades no agravamento e mortalidade pela COVID-19. É provável que o somatório de comorbidades possa atuar em conjunto para facilitar tanto a entrada celular do SARS-CoV-2 mediada pela ACE-2 ${ }^{26}$ nas células quanto favorecer respostas inflamatórias mais agressivas. Estudos sobre esses aspectos são fortemente recomendados.

Mesmo com todos os cuidados metodológicos adotados, este estudo possui limitações: i. A base utilizada é de domínio público e foi construída a partir das fichas de notificação da COVID-19, sem a adequada padronização das variáveis e a ausência de detalhamento das informações (níveis glicêmicos, estágio da obesidade, controle pressórico, dentre outros); ii. Ao longo da pandemia, diferentes formulários de notificação foram sendo implementados, com exclusão e/ou adição de variáveis; e iii. Por se tratar de uma doença nova, sem clareza do rol de sinais/sintomas, é provável que os menos comuns não tenham sido identificados pelos pacientes e registrados, sobretudo no início da pandemia.

\section{Conclusão}

A prevalência de HAS foi superior à prevalência de DM nos indivíduos que foram a óbito por COVID-19. Em idosos, a prevalência foi superior à observada em indivíduos não idosos. Além disso, verificou-se importante acúmulo de comorbidades e fatores de risco. O perfil clínico e epidemiológico foi caracterizado por idosos, sinais/sintomas indicativos de comprometimento respiratório e predomínio de mais de uma comorbidade. Não se observou diferença entre o tempo do início dos primeiros sintomas e o óbito na análise segundo sexo e faixa etária.

Recomendamos estudos que possam estimar o risco de gravidade de acordo com o número e o tipo de comorbidades preexistentes.

\section{Contribuição dos autores}

Concepção e desenho da pesquisa; Obtenção de dados; Análise e interpretação dos dados; Análise estatística; Obtenção de financiamento; Redação do manuscrito e Revisão crítica do manuscrito quanto ao conteúdo intelectual importante: Santos LG, Baggio JAO, Leal TC, Costa FA, Fernandes TRMO, Silva RV, Armstrong A, Carmo RF, Souza CDF

\section{Potencial conflito de interesse}

Não há conflito com o presente artigo.

\section{Fontes de financiamento}

O presente estudo não teve fontes de financiamento externas.

\section{Vinculação acadêmica}

Não há vinculação deste estudo a programas de pósgraduação.

\section{Aprovação ética e consentimento informado}

Este artigo não contém estudos com humanos ou animais realizados por nenhum dos autores. 


\section{Referências}

1. Shereen MA, Khan S, Kazmi A, Bashir N, Siddique R. COVID-19 infection: Origin, transmission, and characteristics of human coronaviruses. J AdvancRes [Internet]. 2020 Mar 16 [cited 2020 Jun 2];24:91-98. DOI doi. org/10.1016/j.jare.2020.03.005. Available from: https://www.sciencedirect. com/science/article/pii/S2090123220300540.

2. Strabelli TMV, Uip DE. COVID-19 e o Coração. Arq Bras Cardiol. [Internet]. 2020 Mar 30 [cited 2020 Jun 2];114(4):598-600. DOI 10.36660/abc.20200209. Available from: https://www.scielo.br/scielo. php? script $=$ sci_arttext\&pid $=$ S0066-782X2020000400598\&lng $=$ pt\&nr $\mathrm{m}=$ iso

3. Organização Mundial da Saúde. OMS.Coronavirus disease (COVID-19) outbreak situation [Internet]. 2020 [cited 2020 Aug 04]. Available from: https://www.who.int/emergencies/diseases/novel-coronavirus-2019?gclid $=$ CjwKCAjwlZf3BRABEiwA8Q0qq-OYAuqVKv-pzn_skILWYn5zVY7IsveG GHw06SzO6rGcXqRkSJZGRoC4_8QAvD_BwE.

4. MonitoraCOVID-19. Painel Brasil [Internet]. 2020 [cited 2020 Jun 10]. Available from: https://bigdata-covid19.icict.fiocruz.br/.

5. Porcheddu R, Serra C, Kelvin D, Kelvin N, Rubino S. Similarity in Case Fatality Rates (CFR) of COVID-19/SARS-COV-2 in Italy and China. J Infect Dev Ctries .[Internet]. 2020[cited 2020 Jun 10];14(2):125-128. Available from: https:// jidc.org/index.php/journal/article/view/32146445.

6. Shereen M A, Suliman K, Kazmi A, Bashir N, Siddique R. COVID-19 infection: Origin, transmission, and characteristics of human coronaviruses. J Adv Res [Internet]. 2020 Jul [cited 2020 Jul 10];24:91-98. Available from: https://www.sciencedirect.com/science/article/pii/S2090123220300540.

7. Richardson S, Hirsch JS, Narasimhan M, Crawford JM, McGinn T, Davidson $\mathrm{KW}$, et al. Presenting Characteristics, Comorbidities, and Outcomes Among 5700 Patients Hospitalized With COVID-19 in the New York City Area. JAMA [Internet]. 2020 May 26 [cited 2020 Jun 8];323(20):2052-2059. Available from: https://jamanetwork.com/journals/jama/fullarticle/2765184.

8. Zhou F, Yu T, Du R, Fan G, Liu Y, Liu Z, et al. Clinical course and risk factors for mortality of adult inpatients with COVID-19 in Wuhan, China: a retrospective cohort study. Lancet [Internet]. 2020 Mar 28 [cited 2020 Jun 2];395(10229):1054-1062. Available from: https://www.thelancet.com/ journals/lancet/article/PIIS0140-6736(20)30566-3/fulltext.

9. Teuwen L-A, Geldhof V, Pasut A, Carmeliet P. COVID-19: the vasculature unleashed. Nat Rev Immunol [Internet]. 2020 May 21 [cited 2020 Jun 14];1-3. Available from: http://www.nature.com/articles/s41577-0200343-0

10. Marson F A L, Ortega M M. COVID-19 in Brazil. Pulmonology [Internet]. 2020 Jul/Aug [cited 2020 Jul 2];26:241-4. Available from: https://www.ncbi. nlm.nih.gov/pmc/articles/PMC7183991/

11. Pernambuco(Estado). Secretaria de Saúde. Centro de Informações Estratégicas de Vigilância em Saúde. Informe Epidemiológico № 156/2020 - Pernambuco. 04 de agosto de 2020. Pernambuco (PE); 2020. [cited 2020 Aug 04]. Available from: https://12ad4c92-89c7-4218-9e110ee136fa4b92.filesusr.com/ugd/3293a8_965059e30c594eac88e6b4f87 2b6c042.pdf

12. Melo SPDC, Cesse EAP, Lira PIC, Rissin A, Cruz RSBLC, Filho MB. Doenças crônicas não transmissíveis e fatores associados em adultos numa área urbana de pobreza do nordeste brasileiro. Cien Saude Colet. 2019;24(8):3159-3168.

13. Carmo RF, Nunes BEBR, Machado MF, Armstrong AC, Souza CDF. Expansion of COVID-19 within Brazil: the importance of highways.J Travel Med.2020;0820.

14. Malta DC, Duncan BB, Schmidt MI, Machado MI, Silva AG, Bernal RTI, et al. Prevalência de diabetes mellitus determinada pela hemoglobina glicada na população adulta brasileira, Pesquisa Nacional de Saúde. Rev Bras Epidemiol [Internet]. 2019 [cited 2020 Jun 21];22(27):1-13. Available from: https:// www.scielo.br/scielo.php?pid $=$ S1415-790X2019000300408\&script $=$ sci arttext\&tlng $=$ pt.
15. Brasil. Ministério da Saúde. Vigitel Brasil 2018: vigilância de fatores de risco e proteção para doenças crônicas por inquérito telefônico: estimativas sobre frequência e distribuição sociodemográfica de fatores de risco e proteção para doenças crônicas nas capitais dos 26 estados brasileiros e no Distrito Federal em 2018. Ministério da Saúde, Brasília; 2019.

16. Cuschieri S, Grech S. COVID-19 and diabetes: The why, the what and the how. J Diabetes Complications. 2020 Sep; 34(9): 107637.

17. Li L, Huang T, Wang Y, Wang Z, Liang Y, Huang T, et al. COVID-19 patients' clinical characteristics, discharge rate, and fatality rate of meta-analysis. J Med Virol [Internet]. 2020 Jun 23 [cited 2020 Jul 11];92(6):577-83. Available from: https://pubmed.ncbi.nlm.nih.gov/32162702/.

18. Souza CDF, Leal TC, Santos LG. Does Existence of Prior Circulatory System Diseases Accelerate Mortality Due to COVID-19? Arq Bras Cardiol [Internet]. 2020 Mai 21 [cited 2020 Jul 24]; 115(1):146-147Available from: http:// publicacoes.cardiol.br/portal/abc/portugues/2020/v11501/pdf/11501026.pdf

19. Zhu N, Zhang D, Wang W, Li X, Yang B, Song J et al. A Novel Coronavirus from Patients with Pneumonia in China, 2019. N Engl J Med [Internet]. 2020[cited 2020 Jun 6]; 382:727-733. Available from: https://www.nejm. org/doi/full/10.1056/nejmoa2001017.

20. Adhikari SP, Meng S, Wu Y, Mao Y, Ye E, Wang Q, et al. Epidemiology, Causes, Clinical Manifestation and Diagnosis, Prevention and Control of Coronavirus Disease (COVID-19) During the Early Outbreak Period: A Scoping Review. Infect Dis Poverty .[Internet]. 2020[cited 2020 Jun 2]; 9(1):1-12. Available from: https://link.springer.com/article/10.1186/s40249-020-00646-x.

21. Ejaz H, Alsrhani A, Zafar A, Javed H, Junaid K Abdalla AE, et al. COVID-19 and comorbidities: Deleterious impact on infected patients. J. Infect. Public Health. 2020 [Internet]. 2020[cited 2020 out 23]; 9(1):1-12. Available from: https://www.sciencedirect.com/science/article/pii/ S1876034120305943\#bib0065

22. Hoffmann M, Kleine-Weber H, Schroeder S, Krüger N, Herrler T, Erichsen S, et al. SARS-CoV-2 Cell Entry Depends on ACE2 and TMPRSS2 and Is Blocked by a Clinically Proven Protease Inhibitor. Cell .[Internet]. 2020 Apr 16[cited 2020 Jun 6];181(2):271-280.e8. Available from: https://www.sciencedirect. com/science/article/pii/S0092867420302294.

23. Zheng YY, Ma YT, Zhang JY, Xie X. COVID-19 and the cardiovascular system. Nat Rev Cardiol. [Internet]. 2020[cited 2020 Jul 1]; 17: 259-260. Available from: https://www.nature.com/articles/s41569-020-0360-5.

24. Teuwen L-A, Geldhof V, Pasut A, Carmeliet P. COVID-19: the vasculature unleashed. Nat Rev Immunol. [Internet]. 2020 May 21 [cited 2020 Jun 14];1-3. Available from: http://www.nature.com/articles/s41577-020-0343-0

25. Kaur R, Kaur M, Singh J. Endothelial dysfunction and platelet hyperactivity in type 2 diabetes mellitus: Molecular insights and therapeutic strategies. Cardiovasc Diabetol. [Internet]. 2018 Aug 31[cited 2020 Jul 1];17(1):121. Available from: https://pubmed.ncbi.nlm.nih.gov/30170601/.

26. Engin A. Endothelial dysfunction in obesity. In: Advances in Experimental Medicine and Biology. Adv Exp Med Biol. [Internet]. 2017[cited 2020 Jul 4]; 960: 345-79. . Available from: https://pubmed.ncbi.nlm.nih. gov/28585207/.

27. Codo AC, Davanzo GG, Monteiro LB, Souza G, Muraro S, Carregari V, et al. Elevated Glucose Levels Favor Sars-Cov-2 Infection and Monocyte Response Through a Hif- $1 \alpha /$ Glycolysis Dependent Axis. SSRN [Internet]. 2020[cited 2020 Jul 8]; 1-32. Available from: https://www.unicamp.br/unicamp/sites/ default/files/2020-05/SSRN-id3606770_Cell\%20Met.pdf.

28. Muniyappa R, Gubbi S. COVID-19 pandemic, coronaviruses, and diabetes mellitus. Am J Physiol Endocrinol Metab. [Internet]. 2020 [cited 2020 Jul 12]; 318:736-741. Available from: https://www.unicamp.br/unicamp/sites/ default/files/2020-05/SSRN-id3606770_Cell\%20Met.pdf.

29. Guan W, Ni Z, Hu Y, Liang W, Ou C, He J, et al. Clinical characteristics of coronavirus disease 2019 in China. N Engl J M. [Internet] 2020 [cited 2020 Aug 04]; 382:1708-1720. Available from: https://pubmed.ncbi.nlm.nih. gov/32109013/ 


\section{Comunicação Breve}

30. U.S. Department of Health and Human Services. The health consequences of smoking: 50 years of progress. A report of the Surgeon General. Atlanta: U.S. Department of Health and Human Services; 2014[cited 2020 Aug 04]. Available from: https://www.cdc.gov/tobacco/data_statistics/sgr/50thanniversary/index.htm
31. Testino G. Are Patients With Alcohol Use Disorders at Increased Risk for Covid-19 Infection? Alcohol Alcohol. 2020 [cited 2020 Aug 04]; 55(4):3446. Available from: https: //academic.oup.com/alcalc/advance-article/ doi/10.1093/alcalc/agaa037/5827422 